\title{
Proposal of Equations for Predicting Post-Farrowing Sow Weight
}

\author{
André Luis Mallmann', Gabriela da Silva Oliveira', José Zacarias Rampi', Felipe Basquera Betiolo', \\ Deivison Pereira Fagundes', Jamil Elias Ghiggi Faccin', Ines Andretta², \\ Rafael da Rosa Ulguim', Ana Paula Gonçalves Mellagi' \& Fernando Pandolfo Bortolozzo'
}

\begin{abstract}
Background: Body condition score is used widely in swine production to ensure adequate nutritional levels in sows during gestation and lactation. However, body condition score is not a gold standard for the estimation of nutritional requirements in sows. Post-farrowing sow body weight assessment might serve as a useful approach for the better adjustment of the nutritional requirements during lactation; however, this approach is time-consuming, requires labor, and might result in detrimental effects on the sow behavior and welfare. The objective of the present study, therefore, was to formulate prediction equations for the estimation of post-farrowing sow weight.

Materials, Methods \& Results: Seven equations were formulated for predicting the post-farrowing sow body weight, by using the data from three databases, which comprised a total 522 sows (434 gilts and 88 multiparous). The sows were weighed on Day 112 of gestation and after farrowing within $12 \mathrm{~h}$. The piglets birth weight was recorded within $24 \mathrm{~h}$ after farrowing. The equations were formulated considering all the parity orders. While formulating the equations, the following five variables were used: pre-farrowing body weight, piglets born, litter weight, the interval between pre-farrowing weighing and farrowing (in days), and the total feed intake between pre-farrowing and post-farrowing weighing. The seven models were compared using the sets of possible predictors through regression with the best subsets procedure (Minitab for Windows, v. 18). Equations (EQ) 1, 2, and 4 were validated with a database comprising 732 sows (parity orders: 1-5). The females were weighed on Day 107 of gestation and within $24 \mathrm{~h}$ after farrowing. The predicted weights estimated by EQ 2 and $4(215.4 \pm 34.3 \mathrm{~kg}$ and $216.7 \pm 34.4 \mathrm{~kg}$, respectively) did not significantly differ from the observed weight $(216.8 \pm 34.6 \mathrm{~kg})[P>0.05]$.

Discussion: Pre-farrowing sow body weight was identified as the main input variable required for the estimation of the post-farrowing sow body weight. Thus, even EQ 1, which contained only this variable, exhibited a high coefficient of determination $\left(R^{2}=0.8707\right)$. However, the $R^{2}$ value kept increasing as more input variables were included in the equation. Equation 2, 4, and 6 included the litter weight variable, and the addition of this variable increased the numerical value of $\mathrm{R}^{2}$ from 0.8707 in EQ 1 to 0.8975 in EQ 2. The EQ 3, 5, and 7 considered the piglets born variable as well, which increased the $\mathrm{R}^{2}$ value from 0.8707 in EQ 1 to 0.9119 in EQ 3. The coefficient of determination did not vary much among the equations; therefore, the selection of the prediction equations depended on data availability, feed management, facility, and the reliability of data collection in each farm. Although EQ 1 demonstrated a greater correlation between the predicted and the observed post-farrowing weight compared to the other equations, the values of error in central tendency and the errors due to disturbances were numerically higher for EQ 1 in comparison to the other two equations (EQ 2 and 4). Therefore, it is suggested that EQ 1 should be used as the last choice for the estimation of post-farrowing sow weight as it presented low trueness and precision, and also because the predicted weight estimated by EQ 1 was statistically lower than the observed weight $(211.67 \pm 33.33 \mathrm{~kg} v s .216 .84 \pm 34.62 \mathrm{~kg} ; P=0.012)$. EQ 4 demonstrated higher trueness and precision; however, it did not differ significantly from EQ 2 and 1. Further analyses are required in order to validate EQ 3, 5, 6, and 7. Among the equations that were predicted as well as validated, the simplest and the easiest equation with satisfactory results for trueness and precision was EQ 2, which is as follows:

Post-farrowing sow weight $(\mathrm{kg})=13.03+(0.93 \times$ pre-farrowing body weight, $\mathrm{kg})+(-1.23 \times$ piglets born, $\mathrm{n})$
\end{abstract}

Keywords: nutrition, sow, equation, post-farrowing, weight.

DOI: $10.22456 / 1679-9216.83867$

${ }^{1}$ Setor de Suínos, Faculdade de Veterinária \& ${ }^{2}$ Laboratório de Ensino Zootécnico, Faculdade de Zootecnia, Universidade Federal do Rio Grande do Sul (UFRGS), Porto Alegre, RS, Brazil. CORRESPONDENCE: F.P. Bortolozzo [fpbortol@ ufrgs.br - Tel.: +55 (51) 3308-6123]. Setor de Suínos, Faculdade de Veterinária, Universidade Federal do Rio Grande do Sul (UFRGS). Av. Bento Gonçalves n. 9090. Bairro Agronomia. CEP 90540-000 Porto Alegre, RS, Brazil. 


\section{INTRODUCTION}

Adequate nutrition programs are essential for maintaining the body condition of sow throughout its lifetime to avoid reproductive losses [12]. Mathematical modeling approaches have been developed to predict the nutritional requirements in sows. These models partition the metabolizable energy into sow maintenance, products of conception, and maternal weight gain [6,7]. When the first and second categories are satisfied, any remaining nutrients may then be deposited into the maternal tissue [14].

Overfeeding sows during pregnancy increases the body fat at farrowing, and is known to be associated with the reduction in the voluntary feed intake during lactation. Furthermore, the feed intake of the lactating sows is often low, compromising milk production as well as the subsequent reproductive cycles [11]. Generally, gilts and sows are fed on the basis of their body condition score. However, the use of this measure is not an accurate method on which the sow feeding programs could be based [15]. Therefore, approaches for nutritional management during the gestation and lactation phases of the animals are crucial for sow performance and development of piglets.

In order to estimate the weight loss during lactation, it is necessary to measure the post-farrowing body weight and the weaning weight of the female. However, obtaining the post-farrowing body weight is a laborious and challenging task, as removing the sows from the farrowing crate might cause pre-weaning mortality [14]. Therefore, the present study was conducted with an objective of formulating prediction equations for the estimation of post-farrowing sow body weight.

\section{MATERIALS AND METHODS}

The present study was designed with a data set of 522 sows obtained from three different trials to predict equations for the estimation of the post-farrowing maternal body weight. All trials were approved by the Animal Ethics Committee of the Federal University of Rio Grande do Sul, under process no. 31653; the trials are described below.

\section{Database I}

This trial study was conducted in a sow farm comprising 2,200 females, located in South Region, Brazil, in the period between December, 2016 and April, 2017 (average, minimum, and maximum tem- peratures during this period: $21.0,11.1$, and $35.6^{\circ} \mathrm{C}$, respectively; average relative humidity: $85.3 \%$ ) which corresponds to summer and early autumn seasons in the southern hemisphere. A total of 274 gilts from two different genetic lines, [(PIC Camborough $\left.{ }^{\circledR}\right)^{1}$ and (DB Genética Suína $\left.\left.\mathrm{DB}^{\circledR}\right)^{2}\right]$, were housed individually (2.10 $\mathrm{m} \times 0.70 \mathrm{~m}$ ), with ad libitum access to water. In all the phases of pregnancy, females were fed with corn and soybean meal [3.2 Mcal ME/kg, $12.4 \%$ crude protein (CP), and $0.60 \%$ standardized ileal digestible (SID) Lysine]. The gilts were weighed individually when they were moved from the gestation to the farrowing room (approximately on Day 112 of pregnancy) within $12 \mathrm{~h}$ after farrowing, using a scale with a precision of $0.5 \mathrm{~kg}$ (model WT1000-LED, Weightech $\left.{ }^{\circledR}\right)^{3}$. Birth weight of the live-born piglets and stillborn piglets were recorded within $12 \mathrm{~h}$ after birth, using a scale with a precision of $1 \mathrm{~g}$.

\section{Database II}

This trial was conducted on a farm located in South Region, Brazil, in the period between January, 2016 and April, 2016 (average, minimum, and maximum temperatures during this period: $20.8,15.5$, and $25.7^{\circ} \mathrm{C}$; average relative humidity: $85.3 \%$ ) which corresponds to summer and early autumn seasons in the southern hemisphere. A total of 107 sows (19 primiparous and 88 multiparous; PIC Camborough ${ }^{\circledR}$ ) were housed individually $(2.20 \mathrm{~m} \times 0.60 \mathrm{~m})$, with $\mathrm{ad}$ libitum access to water. The females were fed on corn and soybean meal (3.25 Mcal ME/kg, 13\% CP, and $0.65 \%$ SID Lysine) throughout pregnancy. The sows were weighed individually when they were moved from the gestation to the farrowing room (approximately on Day 112 of pregnancy) within $12 \mathrm{~h}$ after farrowing, using a scale with a precision of $200 \mathrm{~g}$. The birth weight of the live-born piglets and the stillborn piglets were recorded within $12 \mathrm{~h}$ after farrowing, using a scale with a precision of $1 \mathrm{~g}$.

\section{Database III}

This trial was conducted in a farm located in South Region, Brazil, in the period between January, 2017 and February, 2017 (average, minimum, and maximum temperatures during this period: $21.0,11.1$, and $35.6^{\circ} \mathrm{C}$; average relative humidity: $85.3 \%$ ). Gilts $(\mathrm{n}=141)$ from two different genetic lines, PIC Camborough ${ }^{\circledR}$ and DB Genética Suína $\mathrm{DB}^{\circledR}$, were housed individually $(2.10 \mathrm{~m} \times 0.70 \mathrm{~m})$, with ad libitum access 
to water. The females were fed on corn and soybean meal (3.2 Mcal ME/kg, 12.4\% CP, and 0.60\% SID Lysine) throughout pregnancy. The gilts were weighed individually when they were moved from the gestation to the farrowing room (approximately on Day 112 of pregnancy) within $12 \mathrm{~h}$ after farrowing, using a scale with a precision of $0.5 \mathrm{~kg}$ (model WT1000-LED, Weightech $^{\circledR}$ ). The birth weight of the live-born piglets and the stillborn piglets were recorded within $12 \mathrm{~h}$ after farrowing, using a scale with a precision of $5 \mathrm{~g}$.

\section{Validation database}

The predicted equations formulated using the data from Databases I, II, and III were used to estimate the post-farrowing body weight of sows from another independent database. On the basis of the variables in the equations that matched the variables available in the validation database, only EQ 1, 2, and 4 were evaluated for validation.

The validation database consisted of data from a trial conducted in a sow farm comprising 5200 females, located in the state of Mato Grosso, Brazil (Parallel $14^{\circ}$ ), in the period between January 2011 and May 2011. A total of 732 PIC Camborough ${ }^{\circledR}$ females (parity order: 1-5) were weighed individually when they were moved from the gestation to the farrowing room (approximately on Day 107 of pregnancy) within $24 \mathrm{~h}$ after farrowing. All the females had ad libitum access to water. Throughout pregnancy, the sows were fed on corn and soybean meal based on the NRC recommendation [6,7]. The number of live-born and stillborn piglets was recorded prior to cross-fostering.

\section{Statistical Analysis}

The databases from the three trials used for the formulation of the equations were analyzed using the Minitab $^{\circledR}$ software (Minitab for Windows, v. 18) ${ }^{4}$. The pre-farrowing body weight $(\mathrm{kg})$ and post-farrowing body weight $(\mathrm{kg})$ variables were analyzed using the Grubbs' test to ensure that all the data belonged to a normally distributed population. No outlier observation was identified in this analysis, considering the significance level of $5 \%$. The post-farrowing sow weight $(\mathrm{kg})$ was considered a dependent variable in the following steps. The following independent variables were tested: pre-farrowing body weight $(\mathrm{kg})$, total piglets born (live-born and stillborn, n), piglet birth weight (live-born and stillborn, $\mathrm{kg}$ ), feed intake between pre-farrowing weighing and post-farrowing weighing $(\mathrm{kg})$, and the interval between pre-farrowing weighing and farrowing (days). All possible models were compared using the sets of possible predictors through regression with the best subsets procedure. The independent variables that generated models with the most suitable data adjustment (criteria: adjusted coefficient of determination and Mallows's Cp) were selected in this step and analyzed using ANOVA with the mixed-effects model procedure. In this analysis, weighing day and origin database were used as random effects, while sow genetic line was considered a fixed effect. The inclusion sequence of the co-variables in the models was the same as defined in the last procedure.

In order to perform validation, the observed sow body weights obtained from the validation database and the predicted sow body weights obtained from the prediction equations were submitted to General Linear Model, and the means were compared using Tukey's test at a 5\% significance level. Pearson's correlation between the observed and the predicted values was also analyzed.

The accuracy of each method was evaluated considering the closeness between the measurements obtained by the method and the accepted reference values, and was expressed in terms of trueness and precision. The trueness of a measurement indicated the degree of agreement between the expected value (the predicted weights obtained from the equations in this case) and the reference value (the observed weights from the independent validation database); precision indicated the degree of internal agreement between the independent measurements obtained under a specific set of conditions [3]. In the present study, the accuracy of the equations was analyzed by following the procedure of Theil [13] adapted by Pomar \& Marcoux [10]. Briefly, the mean square of the prediction error (MSPE) was calculated by obtaining the sum of squared forecast errors (the difference between the observed value and the predicted value obtained from each equation) and dividing it by the number of experimental observations. The MSPE was then decomposed into the following: error in central tendency (ECT), error due to regression (ER), and error due to disturbances (ED). ECT was the difference between the average observed weight and the average predicted weight. ER was the error value that would have been obtained if the regression of the post-farrowing predicted weight was in complete 
agreement. ED was the variation in the observed weights, which is not considered in the regression of least squares while predicting the weights using the equations. The greater the sum of ECT and ER, the lower was the trueness of the result. Similarly, the greater the ED value, the lesser was the precision of the result. In the present study, trueness was estimated by the sum of the values of ECT and ER, precision was associated with the ED error, and the overall accuracy was expressed in terms of MSPE.

\section{RESULTS}

A total of 522 females (434 gilts and 88 multiparous) from the set of three databases were included in the formulation of the equations. The average prefarrowing weight (mean \pm SD) was $209.8 \pm 19.9 \mathrm{~kg}$, the average post-farrowing body weight was $188.5 \pm$ $22.6 \mathrm{~kg}$, and the interval between the pre-farrowing weighing and farrowing was of $3.7 \pm 1.6$ days. The mean litter birth weight was $18.1 \pm 3.7 \mathrm{~kg}$, and the number of total piglets born (live-born and stillborn) was $13.5 \pm 3$. 1 . Sow parity ranged between 1 and 4 $(2.6 \pm 1.8)$.

Seven equations were formulated (further details are given in Table 1), each with a different set of variables for the estimation of the post-farrowing sow body weight:

EQ 1: $y=4.30+(0.89 \times b 1)$

EQ 2: $y=13.03+(0.93 \times b 1)+(-1.23 \times b 2)$

EQ 3: $y=13.55+(0.96 \times b 1)+(-1.34 \times b 3)$

EQ 4: $\mathrm{y}=8.45+(0.93 \times \mathrm{b} 1)+(-1.18 \times \mathrm{b} 2)+(1.15 \times \mathrm{b} 4)$

EQ 5: $y=8.74+(0.96 \times b 1)+(-1.32 \times b 3)+(1.24 \times b 4)$

EQ 6: $y=7.85+(0.93 \times b 1)+(-1.18 \times b 2)+(1.37 \times b 4)$ $+(-0.09 \times \mathrm{b} 5)$

EQ 7: $y=7.89+(0.96 \times b 1)+(-1.33 \times b 3)+(1.55 \times b 4)$ $+(-0.13 \times b 5)$

where $\mathrm{y}$, sow post-farrowing body weight, $\mathrm{kg}$;

b1, sow pre-farrowing body weight, kg;

b2, number of total piglets born (piglets born alive and stillborn), n;

b3, litter weight (piglets born alive and stillborn), kg; $\mathrm{b} 4$, interval between pre-farrowing weighing and farrowing, days;

b5, feed intake between pre-farrowing weighing and farrowing, $\mathrm{kg}$.

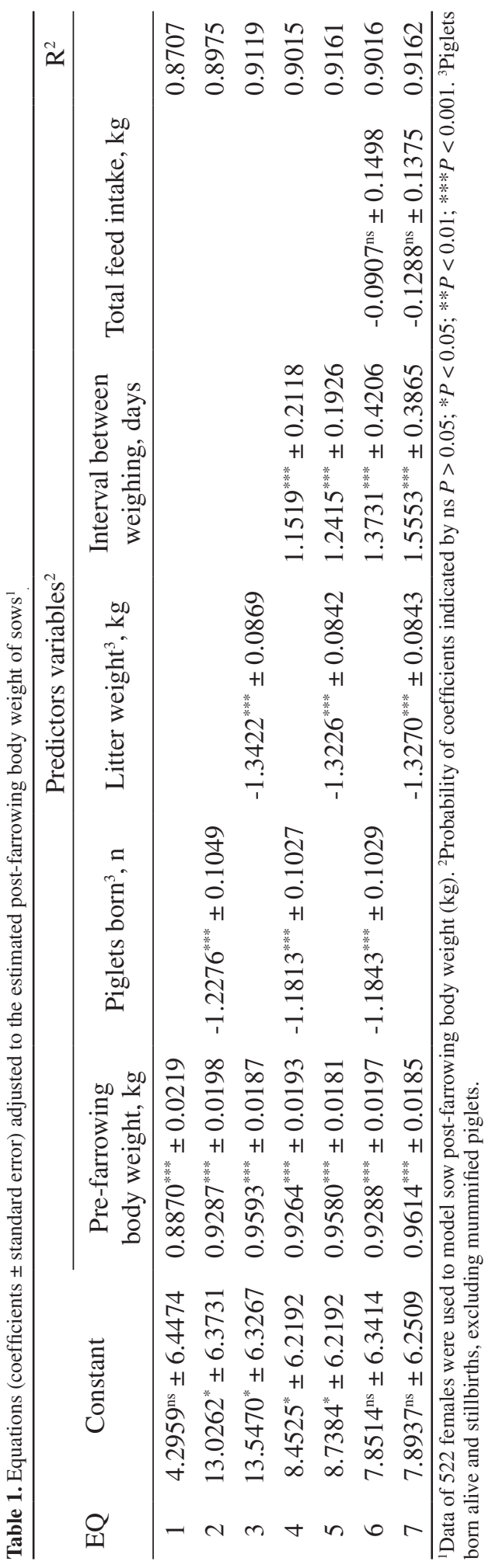


Pre-farrowing body weight, total piglets born (live-born and stillborn piglets), litter weight (liveborn and stillborn piglets), and the interval between pre-farrowing weighing and farrowing were significant $(P<0.001)$ in all the equations. Pre-farrowing body weight was identified as the main input variable for the estimation of the post-farrowing body weight and was included in all the prediction equations. In EQ 2, 3, 4, and 5 , the constant was significant $(P<0.05)$. Total sow feed intake between pre-farrowing and post-farrowing weighing exhibited no significance $(P>0.05)$ in EQ 6 and 7. Since litter weight and total piglets born cor- related $(\mathrm{r}=0.759 ; P<0.001)$, these variables were used separately in the prediction equations.

In the validation trial (Table 2), the average prefarrowing weight (mean $\pm \mathrm{SD}$ ) was $233.8 \pm 37.6 \mathrm{~kg}$, the average post-farrowing body weight was $216.8 \pm 34.6$ $\mathrm{kg}$, and the interval between pre-farrowing weighing and farrowing was of $5.1 \pm 0.9$ days. The number of total piglets born (live-born and stillborn) was $11.9 \pm 3.2$. The predicted weight obtained using EQ 1 was lower than the observed weight $(P=0.012)$; whereas, the predicted weights obtained by using EQ 2 and 4 did not differ from the observed weight obtained from the validation trial.

Table 2. Validation of equations using an independent database ${ }^{1}$.

\begin{tabular}{lcccc}
\hline & Database observed & Equation 1 & Equation 2 & Equation 4 \\
\hline Post-farrowing weight means ${ }^{2}, \mathrm{~kg}$ & $216.84^{\mathrm{a}}$ & $211.67^{\mathrm{b}}$ & $215.42^{\mathrm{ab}}$ & $216.70^{\mathrm{a}}$ \\
Standard deviation, kg & 34.62 & 33.33 & 34.33 & 34.35 \\
Correlation with observed & & 0.95 & 0.96 & 0.96 \\
Error in central tendency & & 26.81 & 2.03 & 0.02 \\
Error due to regression & & 0.07 & 1.25 & 1.28 \\
Error due to disturbances & & 105.51 & 95.48 & 94.91 \\
\hline
\end{tabular}

${ }^{1}$ Data of 732 females were used to validate the equations. ${ }^{2}$ Data submitted to a variance test: $P=0.012$; means with the same letter (a-b) did not statically differ among them by Tukey's test at 5\% level of significance.

\section{DISCUSSION}

Prediction equations could serve as an essential tool in swine industry; however, they are based on input variables, implying that the equation is valid only until the databases are reliable. Prediction equations have been studied and used previously, especially in pork production for the estimation of lean cuts $[1,2]$ and net energy [9]. Recently, an equation was formulated for estimating the post-farrowing sow body weight in order to determine the products of conception (i.e., the fetuses, placenta, and fluids) [14]. In this study, litter weight was not considered as a variable, and parity was considered a fixed effect. Additionally, an intercept variable was created, such that a different constant for each parity had to be introduced into the same equation. In the present study, seven prediction equations were formulated instead of one, in order to better fit the data availability and the demands of each farm. All the equations could be used for all parity orders, and litter weight was included as an input variable. In order to formulate and validate the equations, databases comprising a significant number of animals were used, and the data sets representing the farm reality were carefully collected by the study group.
Among the seven prediction equations formulated in the present study, the first one (EQ 1) was the simplest and easiest for use as it has just one variable. Pre-farrowing sow body weight was identified as the major input variable for estimating the post-farrowing sow body weight; this variable was used in all the seven equations. Even when this input variable was present alone in the equation (EQ 1), the coefficient of determination $\left(R^{2}\right)$ obtained was high $\left(R^{2}=0.8707\right.$ for EQ 1) and close to the $R^{2}$ values obtained for the other equations that contained other input variables as well. However, as depicted in Table 2, these multi-variable equations exhibited a slightly higher $\mathrm{R}^{2}$, which indicated that they were probably more accurate.

In addition to the pre-farrowing body weight, another variable which was common in EQ 2, 4, and 6 was the total piglets born variable. The addition of this variable increased the value of $\mathrm{R}^{2}$ from 0.8707 in EQ 1 to 0.8975 in EQ 2. The increase in the $\mathrm{R}^{2}$ value might be associated with the contribution of piglets to total gravid tissues in late gestation. In early pregnancy, the fetuses correspond for just $8 \%$ of the total gravid uterus; however, as the gestation advances, the uterine tissue increases its participa- 
tion to $40 \%$ at Day 90 of gestation, and to $59 \%$ at Day 110 of gestation [8]. It is possible to obtain the number of piglets born easily without the requirement of qualified labor.

When the interval between pre-farrowing weighing and the farrowing variable was included in the equation in addition to the previous variables, the $\mathrm{R}^{2}$ value increased to 0.9015 (EQ 4). In late gestation, the weight of the fetuses increases cubically, indicating an acceleration in the weight gain in the fetuses during this period [5], as well as in the mammary gland [4] and the female body weight, especially for gilts, which exhibit quadratic increase in weight throughout gestation [4]. Therefore, late gestation is the period of greater weight gain for the females as well as the fetuses. In the prediction equations formulated by Thomas et al. [14], sow body weight gain of $3.1 \mathrm{~kg}$ was predicted for each day in the farrowing crate prior to farrowing; whereas, the model in the present study predicted a per-day increment of $1.19 \mathrm{~kg}$ in the body weight (a value estimated from the descriptive means of this variable in EQ 4 and 5). This difference in the perday increment in the body weight may be attributed to the fact that in the present study, females received the same amount of gestation feed each day during the interval between pre-farrowing weighing and farrowing, and did not have ad libitum access to feed as in the other study. These results support the increase in the $\mathrm{R}^{2}$ value, and highlight the importance of in- cluding the interval between pre-farrowing weighing and farrowing as a variable while formulating the prediction equations.

In EQ 6, the sow feed intake variable was included; however, this variable did not contribute expressively to the increment in the $R^{2}$ value $\left(R^{2}=\right.$ 0.9016). This could probably be explained by no significance of this variable in the equation, as well as by its constant. Besides, obtaining feed intake data is not a simple process, as there are several feed systems, and the process is time-consuming and requires qualified labor. Therefore, this variable may not be considered reliable for the prediction of the post-farrowing sow body weight.

Litter weight was another variable that was common among EQ 3, 5, and 7. All these equations exhibited greater $\mathrm{R}^{2}$ than EQ 2, 4, and 6. However, the impact of total piglets born and litter weight on the prediction equations was reasonably similar. The number of piglets born implicated a decrease of $1.19 \mathrm{~kg}$ per piglet on the post-farrowing body weight (a value estimated from the descriptive means of this variable in EQ 2, 4, and 6); while litter weight implicated a decrease of $1.33 \mathrm{~kg}$ per piglet on the post-farrowing body weight (a value estimated from the descriptive means of this variable in EQ 3, 5, and 7). The coefficients of determination and constants did not vary widely among the equations with the inclusion of the number of piglets born and litter weight variables. This was expected as these two variables were correlated.

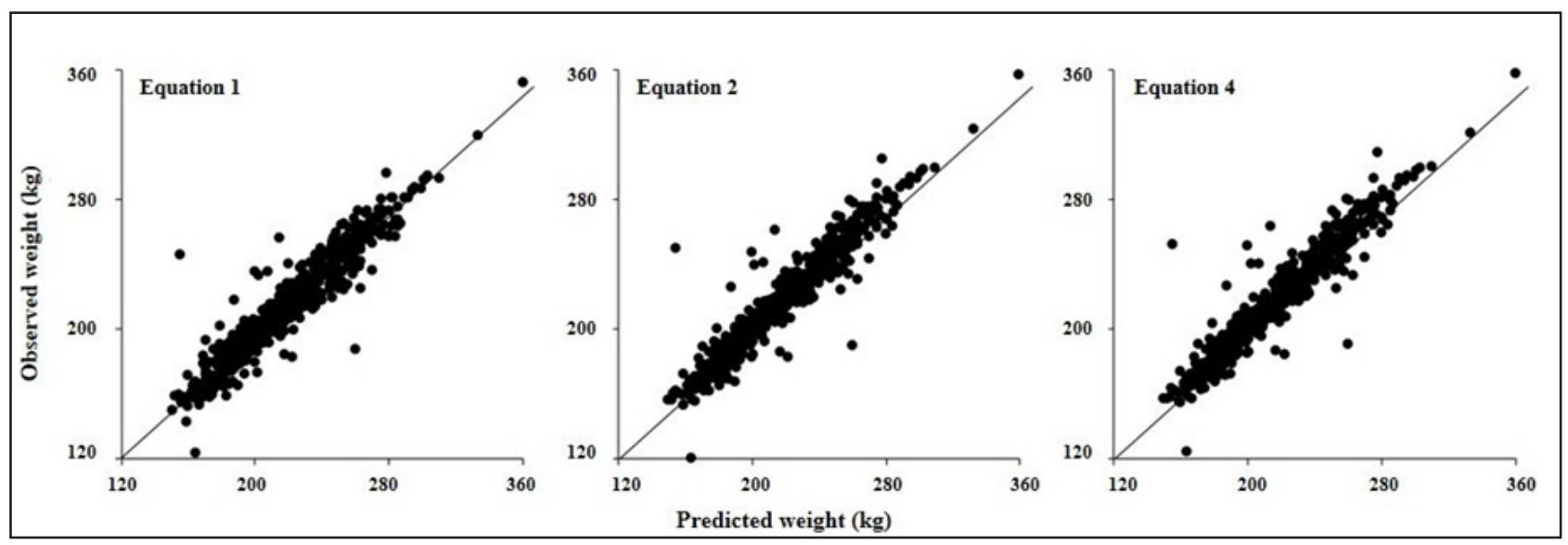

Figure 1. The relationship between observed values from an independent database and values predicted by the equations to the sows post-farrowing body weight. Equations are presented and identified in Table 1. 
As observed in Figure 1, which depicts the validation results, EQ 1, 2, and 4 presented a great correlation between the predicted and observed post-farrowing sow body weights $(0.95,0.96$, and 0.96 , respectively). Besides, ECT and ED decreased with the increase in the number of variables included in the equations. Therefore, EQ1 exhibited higher ECT and ED values compared to EQ2 and EQ4. In other words, EQ 1 exhibited lower trueness (ECT $+\mathrm{ER}$ ) and precision compared to the other equations. This information corroborated the fact that the predicted weight estimated by EQ1 was lower than the observed weight. Thomas et al. [4] demonstrated that the difference of $-1.5 \mathrm{~kg}$ between the observed and the predicted post-farrowing sow body weight did not appear to exhibit biological significance, as it corresponds to a difference of only $32 \mathrm{kcal}$ on the daily sow maintenance requirements, and $0.01 \mathrm{~kg}$ on the expected maternal gain. However, EQ1 exhibited a difference of $5 \mathrm{~kg}$ when a comparison with the observed weight was performed. Therefore, EQ 1 is suggested to be used as the last choice for the estimation of post-farrowing sow body weight, because of its low trueness and precision, and also because the predicted weight estimated by EQ 1 was statistically lower than the observed weight.

Equations 2 and 4 exhibited the same correlation value and a reasonably similar MSPE; thus, both of them could be used for the estimation of postfarrowing sow body weight. As mentioned earlier, the interval between pre-farrowing weighing and farrowing is important for the development of the sow and its conceptus, and then, contributes to increase the $\mathrm{R}^{2}$ value of the equation. In the validation analysis, EQ4 exhibited numerically lower MSPE compared to EQ 1 and 2, indicating the higher accuracy of EQ 4. Therefore, if a farm records data for the interval between pre-farrowing weighing and farrowing, it would be possible to calculate the post-farrowing sow weight using EQ4, and thus, obtain a result with greater correlation. However, among all the formulated prediction equations, EQ 2 was the simplest and easiest for use, with satisfactory results for trueness and precision; therefore, it might be the most suitable choice to introduce this equation to the farms, as the data required for this equation would be easy to collect.

It is important to note that the validation trial was conducted in conditions similar to that in which the trials for the formulation of the prediction equations were conducted, which explains the high correlation between the predicted and observed post-farrowing body weights. However, feed management among farms varies, and this might have been one of the reasons for the high values of ED in all the validated equations. Besides, the equations were validated using just one genetic line. Further studies are required to investigate the interactions of the equations with other genetic lines, and whether the high correlation will persist and the ED would decrease with the use of other genetic lines for validation despite the variations in feed management.

\section{CONCLUSION}

In conclusion, prediction equations based on reliable data could serve as a useful tool for the estimation of post-farrowing sow body weight, eliminating the requirement for removing the female from the farrowing crate and consequently avoiding the piglet mortality. In addition, this tool allows the determination of energy partitioning between the maternal and fetal tissues, thereby allowing the adjustment of the sow nutritional requirements during the subsequent cycle using this information. It is possible that the prediction equations become highly useful in the swine industry, provided that the accuracy of the input variables, which is essential for the adoption of this tool, is ensured.

Further validation analyses are required to validate EQ 3, 5, 6, and 7. Finally, among the predicted as well as validated equations, the simplest and easiest equation with satisfactory results for trueness and precision was EQ 2, which is given below:

Post-farrowing sow weight $(\mathrm{kg})=13.03+$ $(0.93 \times$ pre-farrowing body weight, $\mathrm{kg})+(-1.23 \times$ piglets born, $\mathrm{n}$ )

\section{MANUFACTURERS}

${ }^{1}$ PIC USA Inc. Hendersonville, TN, USA.

${ }^{2}$ Danbred, DB Genética Suína. Patos de Minas, MG, Brazil

${ }^{3}$ Weightech Equipamentos de pesagem, São Paulo, SP, Brazil.

${ }^{4}$ Minitab, Inc. International Sales and Support. State College, PA, USA.

Acknowledgements. The authors are grateful to PIC@ group for the financial support, Master Company for providing the facilities to perform this study and the authors would like to thanks Coordenação de Aperfeiçoamento de Pessoal de Nível Superior (CAPES).

Ethical approval. All trials were approved by the Animal Ethics Committee of the Federal University of Rio Grande do Sul, under process no. 31653 .

Declaration of interest. The authors report no conflict of interest. The authors alone are responsible for the content and writing of the paper. 


\section{REFERENCES}

1 Bereskin B. 1984. Comparing prediction equations to estimate lean cuts in swine. Journal of Animal Science. 59: 1270-1280.

2 Brannaman J.L., Christian L.L., Rothschild M.F. \& Kline E.A. 1984. Prediction equations for estimating lean quantity in 15-to 50-kg Pigs. Journal of Animal Science. 59(4): 991-996.

3 International Organization for Standardization I. 1993. Statistics - Vocabulary and symbols Part 1: Probability and general statistical terms. Geneva: International Organization for Standardization - ISO, 105p.

4 Ji F., Wu G., Blanton J.R. \& Kim S.W. 2005. Changes in weight and composition in various tissues of pregnant gilts and their nutritional implications. Journal of Animal Science. 83(2): 366-375.

5 McPherson R.L., Ji F., Wu G., Blanton J.R. \& Kim S.W. 2004. Growth and compositional changes of fetal tissues in pigs. Journal of Animal Science. 82(9): 2534-2540.

6 National Research Council (NRC). 1998. Nutrient Requirements of Swine. 10th Revised Edition. Washington: National Academy Press, 210p.

7 National Research Council. 2012. Nutrient Requirements of Swine. 11th Revised edition. Washington: National Academy Press, 420p.

8 Noblet J., Close W.H., Heavens R.P. \& Brown D. 1985. Studies on the energy metabolism of the pregnant sow. 1. Uterus and mammary tissue development. British Journal of Nutrition. 53(2): 251-265.

9 Noblet J., Fortune H., Shi X.S. \& Dubois S. 1994. Prediction of net energy value of feeds for growing pigs. Journal of Animal Science. 72(2): 344-354.

10 Pomar C. \& Marcoux M. 2005. The accuracy of measuring backfat and loin muscle thicknesses on pork carcasses by the Hennessy HGP2, Destron PG-100, CGM and ultrasound CVT grading probes. Canadian Journal of Animal Science. 85(4): 481-492.

11 Revell D.K., Williams I.H., Mullan B.P, Ranford J.L. \& Smits R.J. 1998. Body composition at farrowing and nutrition during lactation affect the performance of primiparous sows: I. Voluntary feed intake, weight loss, and plasma metabolites. Journal of Animal Science. 76(7): 1729-1737.

12 Schenkel A.C., Bernardi M.L., Bortolozzo F.P. \& Wentz I. 2010. Body reserve mobilization during lactation in first parity sows and its effect on second litter size. Livestock Science. 132(1): 165-172.

13 Theil H. 1966. Applied Economic Forecasting. Amsterdam: North-Holland Publishing Company, 474p.

14 Thomas L.L., Dritz S.S., Goodband R.D., Tokach M.D., DeRouchey J.M. \& Woodworth J.C. 2016. Generating an Equation to Predict Post-Farrow Maternal Weight in Multiple Parity Sows. In: Kansas Agricultural Experiment Station Research Reports. 2(8): 70-76.

15 Young M.G., Tokach M.D., Goodband R., Nelssen J.L. \& Dritz S.S. 2001. The relationship between body condition score and backfat in gestating sows. In: Kansas Agricultural Experiment Station Research Reports. 10: 5-9. 